\title{
Dynamic Contrast-Enhanced Breast MRI for Evaluating Residual Tumor Size after Neo-Adjuvant Chemotherapy
}

\author{
SHERINE K. AMIN, M.D.; AHMED EL SHIMY, M.D. and MAHMOUD M.A. ABDUL SALAM, M.Sc. \\ The Department of Radiodiagnosis, Faculty of Medicine, Ain Shams University
}

\begin{abstract}
Background: Breast cancer is the most commonly occurring cancer in women and one of the most important causes of death. 1.67 million new cases of breast cancer were identified worldwide, accounting for $25 \%$ of all cancers. The incidence and mortality rates of breast cancer is rising. Patient survival rates depend on both early diagnosis and improved treatment modalities including surgery, radiotherapy and chemotherapy.
\end{abstract}

Aim of Study: To investigate the accuracy of dynamic contrast material-enhanced (DCE) breast MRI for determining residual tumor size after neoadjuvant chemotherapy (NAC) and detect the value of measuring residual size in delayed phase compared to early phase.

Patients and Methods: The study started at December, 2019 and included twenty female patients who were newly diagnosed breast cancers after completion of NAC referred from surgical oncologists to Radiodiagnosis Departments of Ain Shams University and Nasser Institute Hospitals. Each patient was subjected to full history, reviewing medical sheet and dynamic contrast enhanced MRI using $1.5 \mathrm{~T}$ unit (GE).

Results: DCE-MRI after NAC underestimation rate was found to be about $50 \%$ Vs $15 \%$ overestimation rate. $51 \%$ NPV Vs 93\% PPV was reported. Delayed phase better correlated with postsurgical histopathological size than early phase.

Conclusion: DCE-MRI has lower accuracy when performed after NAC. It tends to underestimate residual tumor size. Delayed phase is more accurate than early phase in evaluating residual tumor size.

Key Words: Dynamic contrast-enhanced - Breast MRI-Neoadjuvant chemotherapy.

\section{Introduction}

BREAST cancer is the most commonly occurring cancer in women and one of the most important causes of death. 1.67 million new cases of breast

Correspondence to: Dr. Sherine K. Amin, The Department of Radiodiagnosis, Faculty of Medicine, Ain Shams University cancer were identified worldwide, accounting for $25 \%$ of all cancers. The incidence and mortality rates of breast cancer is rising. Patient survival rates depend on both early diagnosis and improved treatment modalities including surgery, radiotherapy and chemotherapy [1]

Neoadjuvant chemotherapy (NAC) has a wellestablished role in the management of breast cancer. It increases rates of breast-conserving therapy compared with post-operative chemotherapy and may minimize the need for aggressive nodal surgery with axillary lymph node dissection [2] .

The underestimation of residual tumor sizes after NAC can result in positive resection margins and the necessity of re-excisions or even conversions to mastectomies, with the increased risk of subsequent in-breast tumor recurrences, so the accurate assessment of residual tumor size after NAC is crucial for planning the necessary surgical extent, as well as for monitoring the response to NAC [3]

The immediate goal of MRI after NAC is to provide information on the extent of residual disease in the breast to enable surgeons to obtain adequate surgical margins at initial surgery and avoid re-excision, yet MRI accuracy when performed after NAC is lower [4].

At preoperative MRI in patients with breast cancer who do not undergo NAC, tumor size is assessed in the early phase to maximize the contrast of the tumor to the background parenchyma. However, the rate of enhancement of residual cancer after NAC might be delayed because of the antiangiogenic effects of chemotherapy [5]. 
So, Kim et al. [6] suggested that the lesion size at delayed-phase MRI more accurately reflected the residual tumor size and thus, the current standard practice of assessing size in the early phase may not be adequate following NAC, and the delayed phase should be considered in determining the extent of surgery.

\section{Aim of the work:}

To investigate the accuracy of dynamic contrast material-enhanced (DCE) breast MRI for determining residual tumor size after neoadjuvant chemotherapy (NAC) and detect the value of measuring residual size in delayed phase compared to early phase.

\section{Patients and Methods}

\section{Type of study:}

A prospective study was performed on adult patients with histologically proven breast cancer completed NAC.

\section{Study setting:}

The study was conducted at radio-diagnosis departments of Ainshams university hospitals and Nasser Institute Hospital for Research \& Treatment.

Study period: Six months.

Sampling size: Twenty patients.

\section{Patients:}

A number of 20 female patients aged (35-66 years with 48 year-mean) known breast cancer who were referred from Surgical Oncology Department after completing NAC underwent preoperative DCE-MRI at Ain Shams University and Nasser Institute Hospitals.

Patients were selected according to including and excluding criteri:

\section{Inclusion criteria:}

Females who were diagnosed with breast cancers after the completion of NAC and preparing for surgery.

\section{Exclusion criteria:}

- Female patients without available histopathologic information for residual tumor size.

- Female patients with renal impairment.

- Female patients with cardiovascular implanted electronic devices such as cardiac pacemakers or cardioverter defibrillators.
Methods:

The data that was collected included:

\section{1- Preoperative data:}

Patient characteristics:

- Age.

- Menopausal status.

- Tumor histologic type and grade.

- Tumor hormonal receptor status.

- NAC regimen.

MRI study:

- Ethical consideration:

${ }^{\circ}$ The approval of done investigations were obtained from the patients.

${ }^{\circ}$ The study group were informed about nature and purpose of study.

${ }^{\circ}$ Written consent were taken.

${ }^{\circ}$ Ensuring confidentiality.

Study procedures:

- Patient preparation:

${ }^{\circ}$ Fasting for six hours.

${ }^{\circ}$ Serum creatinine level.

${ }^{\circ}$ Insertion of IV cannula on the contralateral side of affected breast.

- Oral prednisolone 2 and 12 hours before contrast injection for patients with history of allergic reaction.

${ }^{\circ} \mathrm{IV}$ injection of Gd-DTPA $(0.1-0.2 \mathrm{mmol} / \mathrm{Kg})$.

\section{Technique of examination:}

All the MRI examinations were performed with patients in the prone position by using a $1.5-\mathrm{T}$ MRI unit with a dedicated breast coil. The protocol consisted of a sagittal T2-weighted fat-suppressed fast spin-echo sequence and an axial T1-weighted fat-suppressed fast spoiled gradient-echo sequence with one pre-contrast and five post-contrast dynamic series at 90, $180240,300,360$ seconds after beginning the intravenous administration of gadobutrol using an automated injector at a dose of 0.1$0.2 \mathrm{mmol}$ per kilogram of body weight and at a rate of $2 \mathrm{~mL} / \mathrm{sec}$, followed by a $20-\mathrm{mL}$ saline flush. Post processing, including subtraction and maximum intensity projection was performed.

\section{MRI data collection:}

- Lesion size at early phase post-contrast.

- Lesion size at early phase post-contrast. 
- Pattern of lesion enhancement (non, focal, NME and mass).

\section{2- Operative data:}

Type of operation (mastectomy, BCS, skin sparing mastectomy).

\section{3-Postoperative data:}

\section{Pathological findings:}

The pathology reports would include the lesion size, pathological type, grade of the tumor and surgical margin (free or involved).

\section{Statistical analysis:}

The maximal diameter of the residual enhancing tumor was measured. Each tumor was measured on early and late phase MR images. MRI findings were analyzed using the Breast Imaging Reporting and Data System. Power of significance was evaluated as follows: a- Probability level ( $p$-value) $\geq 0.05$ : Insignificant, $\mathrm{b}-p$-value $<0.05$ : Significant, c- $p$-value $<0.01$ : Highly significant.

\section{Results}

Of the 20 patients, $12(60 \%)$ were premenopausal and $8(40 \%)$ were postmenopausal with 49 year-median (Table 1).

The mean size of the tumor was $2.25 \mathrm{~cm} \pm 1 \mathrm{~cm}$ at surgical histopathologic examination. The mean tumor size at delayed-phase MRI was larger than that at early-phase MRI $(2.1 \pm 1.3 \mathrm{Vs} 1.5 \pm 1 \mathrm{~cm})$. The tumor sizes at histopathologic examination correlated with those at delayed-phase MRI more than with those at early-phase MRI $(\mathrm{ICC}=0.887)$ at delayed vs $(\mathrm{ICC}=0.375)$ at early phase The size discrepancy was defined as the tumor size at MRI subtracted by the tumor size at histopathologic examination or the tumor size on delayed phase images subtracted by the tumor size on the earlyphase images (Table 2).

The mean size discrepancy of the lobular cancers was greater than that of the ductal cancers $(1.7 \pm 1 \mathrm{~cm}$ versus $0.7 \pm 0.6 \mathrm{~cm}, p=.005)$, which indicates that lobular cancers tends to become larger than ductal cancers on delayed phase MRI. The mean size discrepancy of NME was greater than that of mass $(2.5 \mathrm{~cm}$ versus $0.7 \pm 0.6 \mathrm{~cm}, p=.007)$, which indicates that NME tends to become larger than mass on delayed phase MRI.

Moreover, the mean size discrepancy of masses having speculated margin was greater than those having regular margins $(0.9 \pm 0.63 \mathrm{~cm}$ versus $0.5 \pm$ $0.3 \mathrm{~cm}, p=.007)$, while there was no much difference regarding the shape of masses $(p=0.425)$ which indicates that mass margin is more affecting size discrepancy between early and delayed phases than mass shape (Table 3).
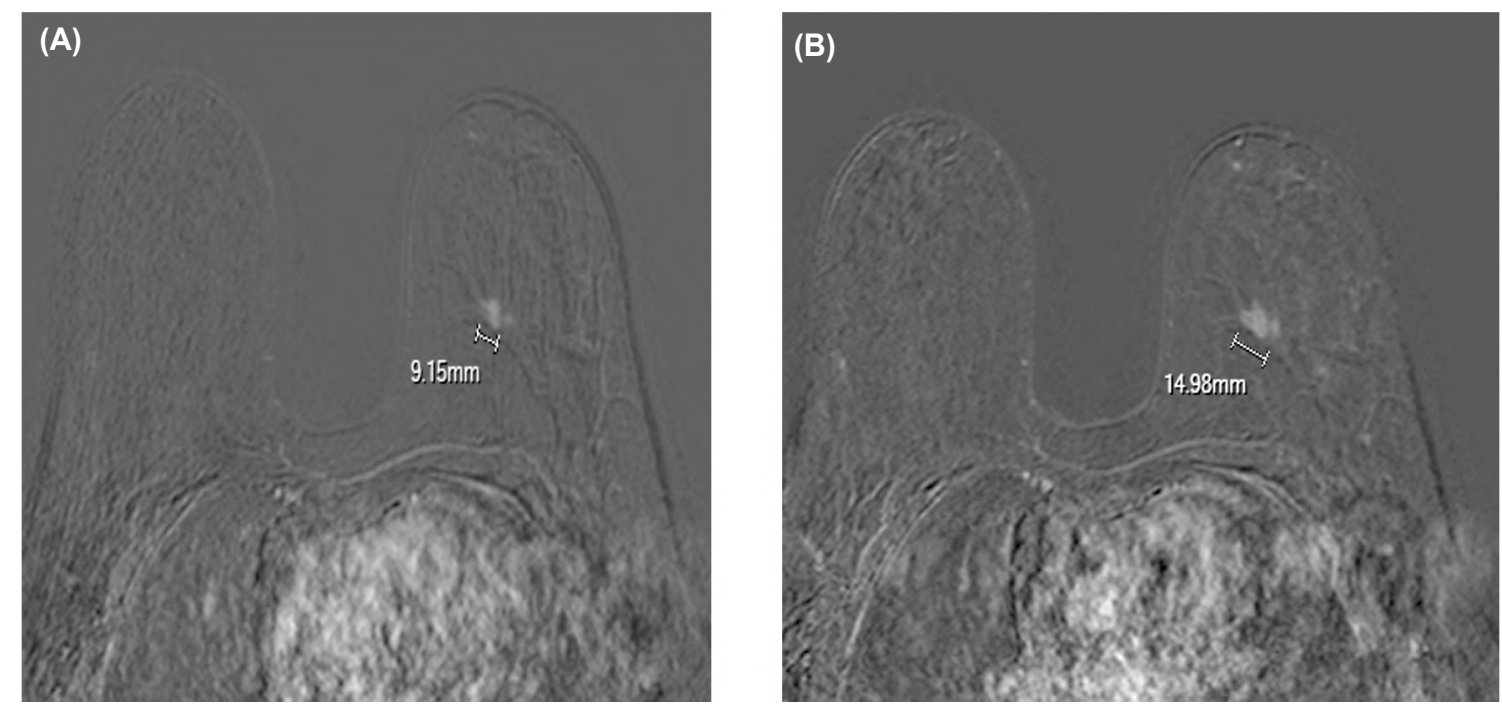

Fig. (1): 35 years old female patient with locally advanced IDC completed six cycles of neo-adjuvant chemotherapy then underwent preoperative DCE-MRI for evaluation of tumor response (a) Axial T1 substracted DCEMRI showed lesion size was $9 \mathrm{~mm}$ on early phase and (B) $15 \mathrm{~mm}$ on late phase. Postoperative Surgical Histopathology revealed IDC with tumor mass measuring $15 \mathrm{~mm}$. 

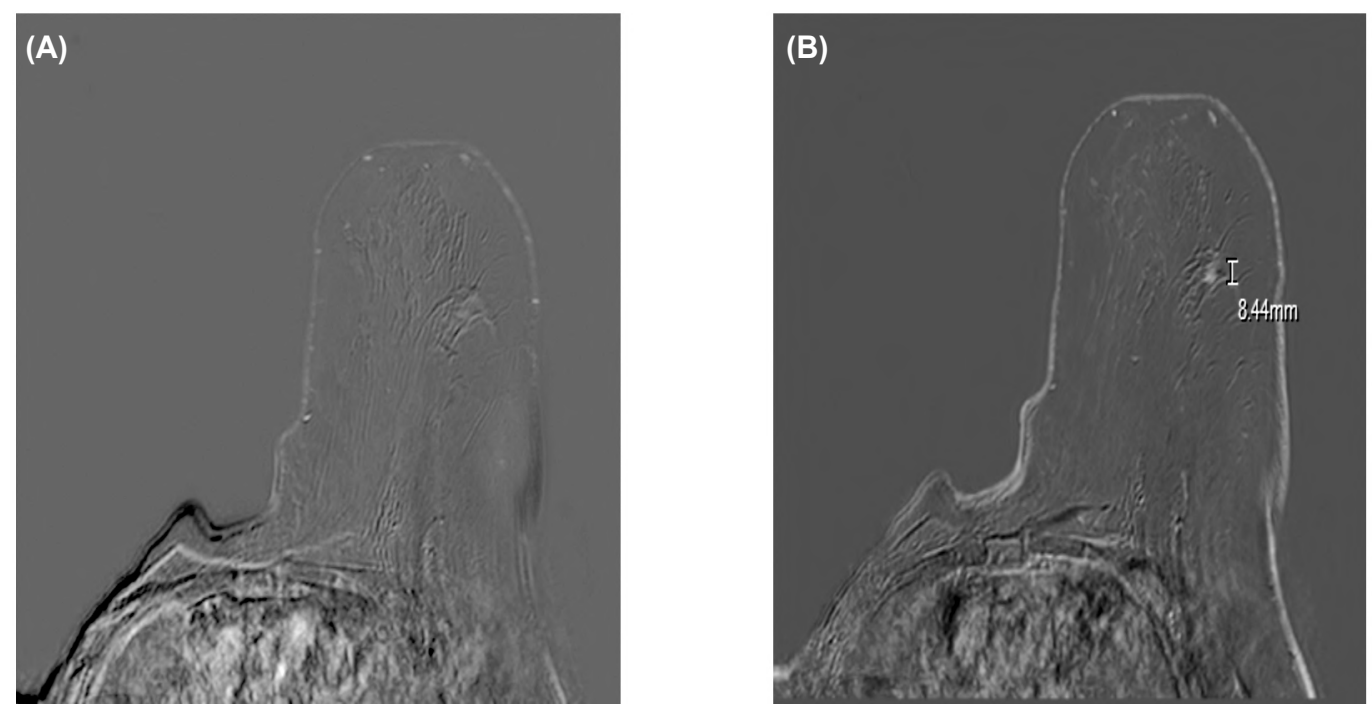

Fig. (2): 60 years old female patient, a known case of right breast cancer was newly diagnosed with left pathologically proven breast cancer completed six cycles of neo-adjuvant chemotherapy then underwent preoperative DCE-MRI for evaluation of tumor response Axial T1 substracted DCE-MRI showed $9 \mathrm{~mm}$ focus on late phase (image B) that was not evident on early phase (image A). Postoperative surgical histopathology revealed bifocal IDC $10 \mathrm{~mm}$ each.

Table (1): Age incidence.

\begin{tabular}{ll}
\hline Characteristic & Datum \\
\hline Mean \pm SD & $48 \pm 9.4$ \\
Median & $49(35-66)$ \\
Premenopausal & $60 \%(12)$ \\
Postmenopausal & $40 \%(8)$ \\
\hline
\end{tabular}

Table (2): Agreement between residual tumor sizes at MRI and histopathologic examination.

\begin{tabular}{lll}
\hline MRI Phase & \multicolumn{1}{c}{ Early } & \multicolumn{1}{c}{ Late } \\
\hline Tumor Size Difference $(\mathrm{cm})$ & $-0.74 \pm 1 \mathrm{~cm}$ & $-0.35 \pm 0.5 \mathrm{~cm}$ \\
Mean Squared Error & 1.48 & 0.70 \\
Interclass Correlation (ICC) & 0.375 & 0.887 \\
\hline
\end{tabular}

Table (3): Factors affecting residual tumor size discrepancy between early phase and delayed phase MRI.

\begin{tabular}{|c|c|c|c|}
\hline Variable & $\begin{array}{l}\text { Size Discrepancy } \\
\quad(\text { Mean } \pm \text { SD })\end{array}$ & Test & $\begin{array}{c}p- \\
\text { value }\end{array}$ \\
\hline \multicolumn{4}{|l|}{ Histologic Type: } \\
\hline Ductal & $0.7 \pm 0.6 \mathrm{~cm}$ & $t=3.853$ & $0.005^{*}$ \\
\hline Lobular & $1.7 \pm 1 \mathrm{~cm}$ & & \\
\hline \multicolumn{4}{|l|}{ Lesion Type: } \\
\hline NME & $2.5 \mathrm{~cm}$ & \multicolumn{2}{|c|}{$\mathrm{F}=4.0040 .007 *$} \\
\hline Focus & $0.6 \mathrm{~cm}$ & & \\
\hline Mass & $0.7 \pm 0.6 \mathrm{~cm}$ & & \\
\hline \multicolumn{4}{|l|}{ Shape: } \\
\hline Rounded to oval & $0.6 \pm 0.05 \mathrm{~cm}$ & \multicolumn{2}{|c|}{$t=0.8260 .425$} \\
\hline Irregular & $0.8 \pm 0.53 \mathrm{~cm}$ & & \\
\hline \multicolumn{4}{|l|}{ Margin: } \\
\hline Regular & $0.5 \pm 0.3 \mathrm{~cm}$ & \multirow{2}{*}{\multicolumn{2}{|c|}{$t=2.5630 .014^{*}$}} \\
\hline Irregular/Spiculated & $0.9 \pm 0.63 \mathrm{~cm}$ & & \\
\hline \multicolumn{4}{|l|}{ Enhancement: } \\
\hline Homogenous/Rim & $0.55 \pm 0.2 \mathrm{~cm}$ & \multicolumn{2}{|c|}{$t=1.5530 .128$} \\
\hline Heterogenous & $0.75 \pm 0.54 \mathrm{~cm}$ & & \\
\hline
\end{tabular}

\section{Discussion}

The accurate assessment of residual size after NAC is crucial from the surgical point of view. The type of surgical intervention whether mastectomy or BCS depends at first place on tumor size relative to breast size and extent of cancer.

The underestimation of residual tumor size increases the rate of positive margins and the overestimation of residual tumor size may affect the cosmetic outcome if the tissue removal is very extensive [7].

According to Jeevan et al. [8] one in five patients who had BCS in England had reoperation. Reoperation after breast conserving surgery has various undesirable consequences. It may delay adjuvant treatments, and some evidence suggests that it is associated with increased rates of local and distant recurrence. It is likely to cause a significantly poorer cosmetic outcome, regardless of whether the reoperation is a second breast conserving procedure or a mastectomy.

Being the most accurate modality in assessing the residual tumor size after NAC, we searched MRI accuracy regarding the accurate size assessment and predicting the pathological complete response.

We found that NAC affects MRI accuracy and tends to underestimate the tumor size in comparison to post surgical histopathology.

The underestimation rate in our study was estimated to be about $50 \%$ versus $15 \%$ overestimation rate. 
Similarly, Hyton [3] stated that MRI when performed after NAC, its accuracy is lower. Also Wu et al. [9] reported the overestimation rate and underestimation rate of residual size after NAC were $25 \%$ and $35 \%$ respectively.

Moreover, we observed that NPV of MRI is much lower than PPV (50\% Vs 93\%) which means that negative post NAC MRI does not necessarily confirm pCR.

Similarly, Bouzon et al. [7] reported $60 \%$ NPV versus $85 \%$ PPV.

The high underestimation rate of MRI leads to increased risk of positive resection margins in breast conserving surgeries and need for futher completion mastectomy, yet we found that the underestimation rate can be improved by measuring the residual size on delayed phase.

Comparing the residual size in early and late phase with histopathology yields better results as regard the delayed phase. We found residual tumor size at delayed phase better correlates with histopathological size than early phase, a finding which would improve the chance of breast conservation.

Kim et al. [6] also concluded that delayed-phase MRI is more accurate than early-phase MRI for evaluating residual breast tumor size after neoadjuvant chemotherapy.

We searched the factors affecting the disconcordance between MRI phases and histopathology and found that tumor histologic type in addition to mass criteria on MRI make difference. Lobular cancer was observed to have higher disconcordance than ductal one and masses showing ill defined speculated margins and heterogenous enhancement have higher early-late phase mismatch than those with regular well-defined margins and homogenous/rim enhancement.

Lower MRI accuracy after NAC can be attributed to the effect of NAC on tumor vascularity leading to disruption of the ordinary enhancement kinetics of malignant lesions.

The fibrous granulation tissue after NAC may contain numerous small vessels and inflammatory cells, it is most likely that these structures mimic the remnant tumor in the MRI findings leading to overestimation or false positive results [10].

The high underestimation rate of the post-NAC MRI for predicting residual disease might have resulted from the microscopically scattered tumor foci and loss of tumor cellularity after NAC. In addition, the reduction of enhancement secondary to the changes in tumor vascularization induced by the anti-angiogenetic effect of chemotherapeutic agents also contributed to the underestimation of post-NAC MRI in the prediction of residual tumor size [9].

The better correlation of late phase than early phase may be explained by decreased tumor vascularity after NAC. Malignant tumors without chemotherapy effect show early enhancement then washout, yet after antiangiogenic effect of chemotherapy cumulative contrast uptake leads to more enhancement at delayed phase giving better prediction of residual size.

Similarly, Schrading et al. [11] noted that the rate of enhancement of residual cancer after NAC might be delayed due to the antiangiogenic effects of chemotherapy.

The MRI diagnostic accuracy (the degree of compatibility of MRI findings whether positive or negative with histopathology) was $80 \%$ compared to $76.09 \%$ according to Acea et al. [12] and $71.1 \%$ according to Bouzon et al. [7].

The overall sensitivity of MRI (the ability to detect residual cancer) was estimated to be $93 \%$ ( $90 \%$ for early phase Vs $98 \%$ for late phase), while the overall specificity (the ability to detect pathological complete response) was estimated to be $51 \%$ (35\% for early phase vs $65 \%$ for late phase).

According to Kim et al. [6], the sensitivity of early and late phases and specificity of early and late phases were $86 \%, 96 \%, 64 \%$ and $40 \%$ respectively.

\section{Surgical application:}

Many women seek BCS rather than MRM due to its better cosmetic outcome. Many studies following thousands of women for more than 20 years showed that when BCS is done along with radiation, the outcome is the same as having a mastectomy, yet loco-regional recurrence is what hinders BCS.

Positive resection margin is the most important factor affecting probability of loco-regional recurrence after BCS, so achieving adequate surgical margin is mandatory.

According to our results, early phase tends to underestimate residual tumor size $(-0.74 \pm 1 \mathrm{~cm}$ discrepancy), so the current protocol of assessing tumor size at early phase may increase the risk of 
positive margins and hence loco-regional recurrence.

So, measuring tumor size at late phase $(-0.35 \pm$ $0.5 \mathrm{~cm}$ discrepancy) would decrease the probability of positive resection margins and loco-regional recurrence. More-over it would decrease the amount of resected breast tissue yielding better cosmetic outcome [13]

\section{Future research:}

There is growing interest toward skipping breast surgery for tumors that have been completely eradicated by NAC. However, complete response can not be confirmed by MRI as we have mentioned before due to low NPV of MRI.

So there are ongoing trials toward canceling surgery by combining MRI negative results with image-guided biopsy of tumor bed.

And this is what Hyton [3] noted. It mentioned that MRI is not likely to be used alone to confirm pCR presurgically and may need to be used in combination with biopsy, particularly if omission of surgery is being considered.

Mann [4] also stated that current trials selecting patients for percutaneous biopsy to omit surgery. Initial results show that percutaneous biopsy increases the NPV of MRI in the diagnosis of pCR to $94.4 \%$, thus rendering this approach potentially possible.

This would be a promising advance in management of breast cancer obviating the burden of surgical intervention.

\section{Conclusion:}

MRI when performed after NAC its accuracy is affected. Post-NAC MRI may underestimate or overestimate the residual size with higher underestimation rate. Delayed phase of DCE-MRI is more accurate than early phase and better correlate with post surgical histopathology, an observation that can improve the underestimation and surgical outcome.

Further trials are needed to search accuracy of targeted biopsy of tumor bed in combination with MRI to confirm complete eradication of tumor after NAC.

\section{References}

1- MOMENIMOVAHED Z. and SALEHINIYA H.: Epidemiological characteristics and risk factors for breast cancer in the world. Breast Cancer (Dovepress), 11: 151-164, 2019.

2- PILEWSKIE M. and MORROW M.: Axillary Nodal Management Following Neoadjuvant Chemotherapy. JAMA Oncol., 3 (4): 549-555, 2017.

3- HYTON N.: Residual Disease after Neoadjuvant Therapy for Breast Cancer: Can MRI Help?. Radiology, 289 (2): 335-336, 2018.

4- MANN R.M., CHO N., MOY L., et al.: Breast MRI: State of the art. Radiology, 292 (3): 520-36, 2019.

5- SANTAMARíA G., BARGALLÓ X., FERNÁNDEZ P.L., et al.: Neoadjuvant systemic therapy in breast cancer: association of contrast-enhanced MR imaging findings, diffusion-weighted imaging findings, and tumor subtype with tumor response. Radiology, 283 (3): 663-72, 2017.

6- KIM S.Y., CHO N., PARK I.A., et al.: Dynamic Contrastenhanced Breast MRI for Evaluating Residual Tumor Size after Neoadjuvant Chemotherapy. Radiology, 289, 2018.

7- BOUZON A., IGLESIAS A., ACEA B., et al.: Evaluation of MRI accuracy after primary systemic therapy in breast cancer patients considering tumor biology: Optimizing the surgical planning, 53 (2): 171-177, 2019.

8- JEEVAN R., CROMWELL D., TRIVELLA M., et al.: Reoperation rates after breast conserving surgery for breast cancer among women in England: Retrospective study of hospital episode statistics. BMJ, 345: e4505, 2012 .

9- WU W., WU H., CHEN C., et al.: Higher underestimation of tumour size post-neoadjuvant chemotherapy with breast magnetic resonance imaging (MRI)-A concordance comparison cohort analysis. PLoS One, 14 (10): e0222917. 2019.

10- FUKUDA T., HORII R., GOMI N., et al.: Accuracy of magnetic resonance imaging for predicting pathological complete response of breast cancer after neoadjuvant chemotherapy: Association with breast cancer subtype. Springerplus, 5: 152, 2016.

11-SCHRADING S. and KUHL C.K.: Breast cancer: Influence of taxanes on response assessment with dynamic contrastenhanced MR imaging. Radiology, 277 (3): 687-696, 2015.

12- ACEA B., BOUZON A., SOLER R., et al.: Diagnostic accuracy of MRI to evaluate tumor response and residual tumor size after neoadjuvant chemotherapy in breast cancer patients. Radiology and Oncology, 50 (9), 2016.

13- KOSLOW S. and SIMMONS R.: Principles of surgical management of invasive breast camcer in John R., Gui G., Tuttle T., Early Breast Cancer from screening to multidisciplinary management. 3 rd edition, CRC Press, pp. 360-364, 2013. 


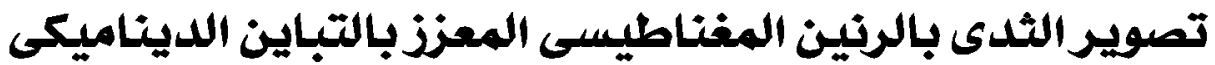

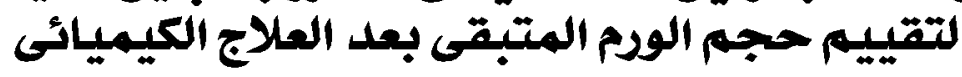

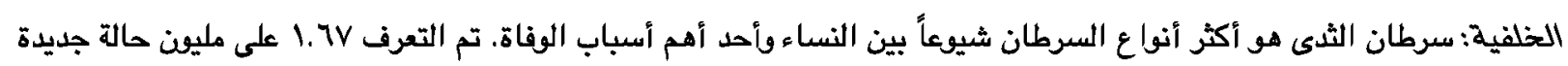

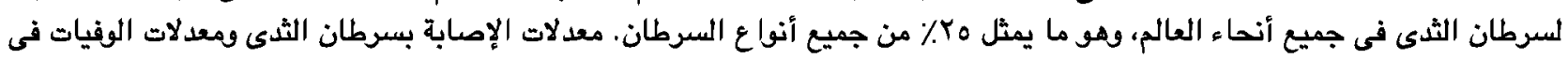

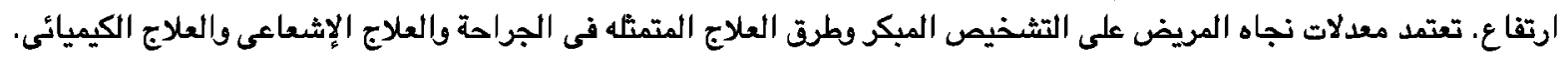

الأهداف: قياس مدى دقة تصوير الثدى بالرنين المغناطيسى بالصبغة فى تصديد حجم الودم المتبقى بعد العلاج الكيميائى الاستباقى

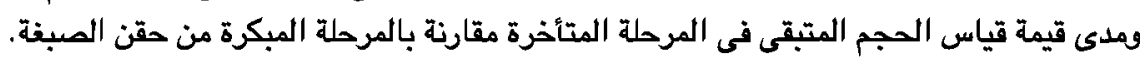

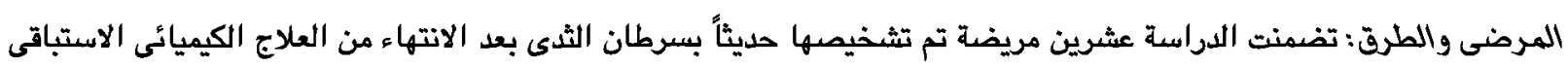

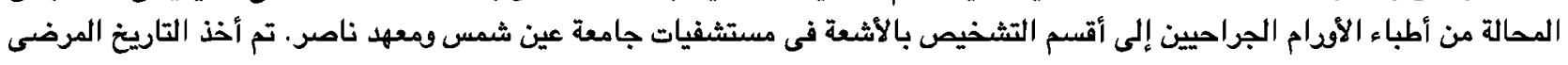

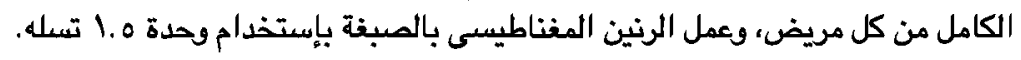

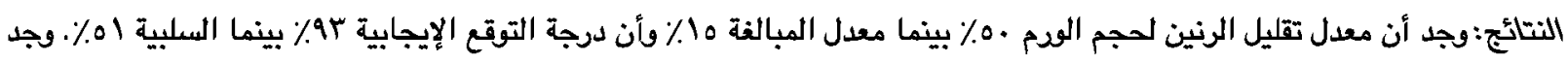

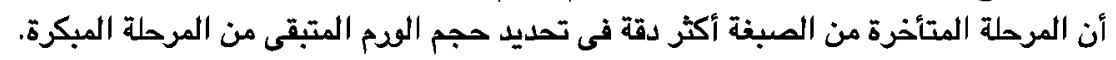

الخلاصة: تتائر دقة الرنين المغناطيسى بالصبغة عند عمله بعد العلاج الكيميائى الاستباقى حيث يميل إلى التقليل من حجم الودم المتبقى.

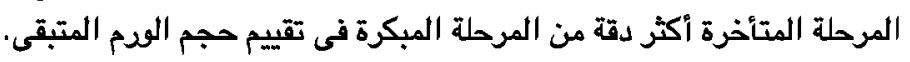

